\title{
Acute ocular chemical injury: a descriptive assessment and management review at St. John Eye Hospital, Jerusalem, Palestine
}

\author{
Riyad Banayot ${ }^{0}$, Yahya Swaiti, Islam Al-hashash
}

St. John Eye Hospital, Jerusalem, Palestine

\begin{abstract}
BACKGROUND: The purpose of the study was to assess caregivers' compliance with the management protocol for chemical injury at St. John Eye Hospital, Jerusalem.

MATERIAL AND METHODS: Charts of all new chemical injury patients who presented to St. John Eye Hospital, Jerusalem, between January and December 2019 were retrospectively reviewed. Data categories collected included: Presentation, age, sex, injury, irrigation, lids, visual acuity, slit-lamp examination (SLE), management plan, and medications given. Data were stored and analysed using Excel.

RESULTS: Patients' presentation date and time, sex, and age were recorded in over $90 \%$ of cases. The mechanism of injury and type of offending chemical were recorded in $65 \%$ of cases. The irrigating solution was identified in $50 \%$ of cases. Corrected visual acuity was recorded in both eyes in almost $50 \%$ of cases. Limbal ischemia was documented in $45 \%$ of cases, and intraocular pressure (IOP) was recorded in $25 \%$. The management plan and explanation of the condition to patients were documented in less than $50 \%$ of cases. Antibiotics and steroids (drops/ointment) were prescribed in $92.5 \%$ of cases.
\end{abstract}

CONCLUSIONS: The results of this study reveal that our documentation needs improvement for several parameters. Several recommendations were formulated:

1. Emphasize to caregivers that irrigation must be done first.

2. Corrected visual acuity should be attempted for both eyes in all cases, and reasons for not recording it should be documented.

3. It is important to document and record limbus details, iris details, and IOP in all cases.

KEY WORDS: chemical injury; protocol; assessment, management, Palestine

Ophthalmol J 2021; Vol. 6, 171-177

\section{INTRODUCTION}

Alkali or acid chemical injury of the conjunctiva and cornea is a severe ophthalmic emergency and needs immediate attention. Chemical injuries can cause extensive damage to the anterior segment of the eye and lead to visual loss and deformity. The severity of chemical eye damage is related to the type of chemical, the volume and concentration 
$(\mathrm{pH})$ of the solution, and the duration of chemical exposure [1]. This potentially blinding condition needs early detection and treatment to secure the best possible outcome.

Immediate irrigation is of utmost importance after chemical or thermal burns until the $\mathrm{pH}$ of the ocular surface is normalized [2-4].

A complete ophthalmic examination (which includes visual acuity) follows irrigation and is used to evaluate the extent and depth of injury. At St. John Eye Hospital (SJEH), we use the Roper-Hall classification [5], which is based on the degree of corneal involvement and limbal ischemia.

During the initial examination, the palpebral fissures should be assessed, and the fornices should be cleaned. Trapped particulate matter can cause permanent damage, even with irrigation. The intraocular pressure (IOP) should also be documented, as alkali injuries have been found to cause acute and chronic elevation of IOP [6].

Most authorities recommend a graded approach depending on the severity of the injury. Mild burns (Roper-Hall grade I and II) respond well to medical treatments, which include topical antibiotic ointment, topical cycloplegics, artificial tears, and steroid drops. In more severe burns, more intensive medical therapies and surgery is necessary [7].

According to McCulley, the clinical course of ocular chemical injury can be classified into four phases: immediate, acute, early reparative, and late reparative [8].

The immediate management protocol adopted at SJEH, which reflects the protocols published by the American Academy of Ophthalmology [9] and Royal College of Ophthalmologists [10], is as follows (done for both eyes):

1 - topical anesthesia;

2 - lid retraction using hooks or eye retractors (Desmarres);

3 - irrigation with at least $1 \mathrm{~L}$ of saline;

4 - check $\mathrm{pH}$ regularly with universal indicator paper until a $\mathrm{pH}$ of 7.0 is achieved;

5 - document age, sex, patient's presenting date and time, attending caregiver presenting time, injury (which eye), mechanism, place and time of injury, offending chemical, irrigating solution type, amount and duration of irrigation, and $\mathrm{pH}$;

6 - perform eyelid eversion and double eversion and remove the offending material;

7 - perform debridement;
8 - document lid eversion and debridement. The attending physician must sign the examination sheet.

9 - take visual acuity (VA) for both eyes (presenting and corrected using pinhole). Document VA for both eyes and reason for not measuring VA. The attending caregiver must sign the examination sheet;

10 - perform a slit-lamp examination (SLE) and document degree of corneal, conjunctival, limbal involvement, intraocular pressure, lid injuries, and lens status. Attending physicians must sign the examination sheet;

11 - document the grade of injury according to Roper-Hall (modified Hughes) classification, explanation of the condition to the patient, plan of action, and follow-up. The attending physician must sign the examination sheet.

12 - document treatment prescribed (according to the type of the offending agent (acid or alkali) and grade of injury. Document the advice given to the patient.

The purpose of the study was to determine whether the procedures implemented for chemical injury patients attending St. John Eye Hospital (SJEH) by our caregivers and physicians during the immediate phase follow accepted standards and protocol and whether those procedures were documented. Data collection included: immediate management, injury, irrigation, debridement, visual acuity, SLE, management and medication, proper documentation of all procedures done, and signatures. Figure 1 shows the chemical injury protocol adopted by the SJEH governance committee and is placed in all the hospital clinics. All practising ophthalmologists and caregivers at the hospital are encouraged to follow the protocol for all chemical injury patients.

\section{MATERIAL AND METHODS Design}

A cross-sectional descriptive observational design was used to evaluate compliance with chemical injury protocol at SJEH, Jerusalem, Palestine.

The charts of all new chemical injury patients who presented to the Emergency Department at SJEH, Palestine, between January and December 2019 were retrospectively reviewed. The total number seen was 40 patients. Ethical approval for the study was obtained from SJEH Ethics Committee. The confidentiality of the study was maintained 


\section{CHEMICAL INJURY PROTOCOL}

Ophthalmic Chemical injury is an ophthalmic EMERGENCY.

Almost any chemical can cause ocular irritation; serious damage generally results from either strongly basic (alkaline) compounds or acidic especially in regard to poor immediate treatment, proper documenta It is important to adhere to the protocol.

HISTORY: Must record name of substance, whether particulate or liquid, if work-related, the time of injury, the time of attendance to eye hospital, and if irrigated before attendance.

\begin{tabular}{|l|l|l|l|l|}
\hline EXAMINATION & Grade I & Grade II & Grade III & Opaqu IV \\
\hline Cornea & No opacity & Hazy & Obscure details & No view \\
\hline Iris details and pupil & visible & Visible & Ischemia 1/3 $-1 / 2$ & Ischemia $>1 / 2$ \\
\hline Limbus & No ischemia & Ischemia < 1/3 & No ischemia & Ischemic necrosis \\
\hline Proximal conj \& sclera & No ischemia & No ischemia & Guarded & Dismal \\
\hline Prognosis & Excellent & Good & \multicolumn{2}{|l}{} \\
\hline
\end{tabular}

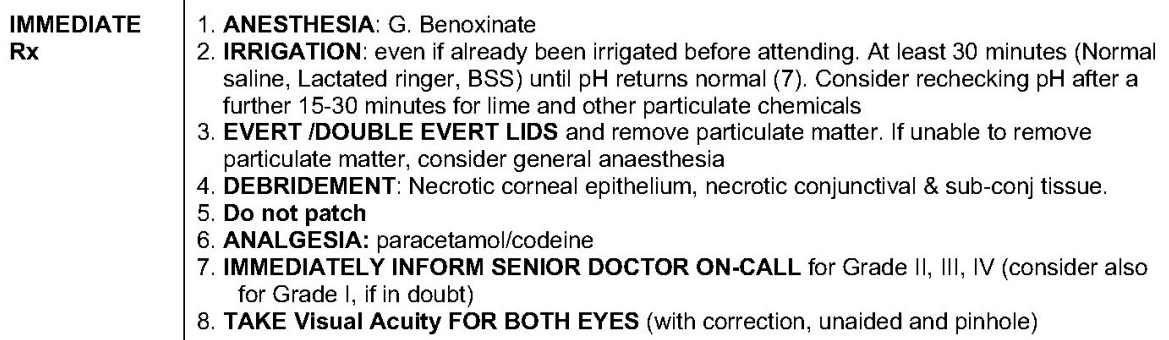

3. EVERT IDOUBLE EVERT LIDS and remove particulate matter. If unable to remove particulate matter, consider general anaesthesia

4. DEBRIDEMENT: Necrotic corneal epithelium, necrotic conjunctival \& sub-conj tissue

5. Do not patch

6. ANALGESIA: paracetamol/codeine

7. IMMEDIATELY INFORM SENIOR DOCTOR ON-CALL for Grade II, III, IV (consider also for Grade I, if in doubt)

8. TAKE Visual Acuity FOR BOTH EYES (with correction, unaided and pinhole)

\begin{tabular}{|c|c|c|}
\hline Silt lamp & GRADE I & GRADE II / III / IV \\
\hline $\begin{array}{l}\text { ACUTE } \\
\text { PHASE } \\
\text { (DAY } 0 \text { - 7) } \\
\text { Do not use } \\
\text { Vit. C in non- } \\
\text { alkali burns }\end{array}$ & $\begin{array}{l}\text { - G. Pred Forte X } 8 \\
\text { - Consider Oral Diamox } \\
250 \mathrm{mg} \times 4 \text { if IOP } \\
\text { difficult to take } \\
\text { - G. Ofloxacin X } 4 \\
\text { - G. Cyclopentolate X } 3 \\
\text { - G. Blink X } 8\end{array}$ & $\begin{array}{l}\text { - G. Pred Forte } \times 8 \\
\text { - Oral Diamox } 250 \mathrm{mg} \times 4 ; \pm \text { G. Timolol } 0.5 \% \times 2 \\
\text { - G. Cyclopentolate } \times 3 \\
\text { - G. Blink } \times 8 \\
\text { - Topical Vit. C } 10 \% \times 6 \\
\text { - Oral Vit. C } 2 \mathrm{Gm} / \mathrm{d} \\
\text { - Oc. Tetracycline } \times 4 \\
\text { - Doxycycline } 100 \mathrm{mg} \times 2 \\
\text { - Consider rodding (if symblepharon likely) }\end{array}$ \\
\hline $\begin{array}{l}\text { EARLY } \\
\text { REPAIR } \\
\text { (DAY } 7-21 \text { ) }\end{array}$ & $\begin{array}{l}\text { Complete re-epithelisation } \\
\text { - Stop G. Cyclopentolate } \\
\text { - Decrease / stop Diamox } \\
\text { - Decrease + stop steroids by } \\
\text { day } 10 \\
\text { - Decrease / stop antibiotics } \\
\text { - G. Blink X } 6\end{array}$ & $\begin{array}{l}\text { Fallure or partial failure to re-epithelise } \\
\text { - Stop G. Cyclopentolate } \\
\text { - Decrease / stop Diamox } \\
\text { - Decrease + stop steroids by day } 10 \\
\text { - Decrease + stop local and systemic antibiotics } \\
\text { - Decrease + stop local and systemic Vit. C (except Grade IV) } \\
\text { - G. Voltaren } 0.1 \% \times 4 \\
\text { - G. Blink X } 6\end{array}$ \\
\hline $\begin{array}{l}\text { LATE REPAIR } \\
(\text { DAY }>21)\end{array}$ & - Decrease + Stop G. Blink & $\begin{array}{l}\text { - G. Voltaren } 0.1 \% \times 4 \\
\text { - G. Blink X } 6 \text { for long periods } \\
\text { - Surgical options in cases of absent re-epithelization: } \\
\text { - Stem-cell transplant, followed by PK } \\
\text { - Amniotic membrane graft }\end{array}$ \\
\hline $\begin{array}{l}-C_{y} \\
-D \\
-S t\end{array}$ & & $\begin{array}{l}\text { ler } 3 \text { months) } \\
\text { on) } \\
\text { and spacer when difficult to remove chemical from conjunctiva) } \\
\text { t) }\end{array}$ \\
\hline
\end{tabular}

FIGURE 1. Chemical injury protocol

by masking the names of patients. No patient with chemical injury was excluded from the study.

\section{Dataset synthesis and analysis}

A structured questionnaire form (Fig. 2) was developed as a research tool to collect data. Data categories collected included:
- presentation: time and date, the physician attending time, age and sex of patient;

- injury: which eye, mechanism of injury, time and place, type of chemical;

- actions taken first: irrigation or visual acuity;

- irrigation: type of solution, amount, duration, $\mathrm{pH}$, a signature of irrigating person; 


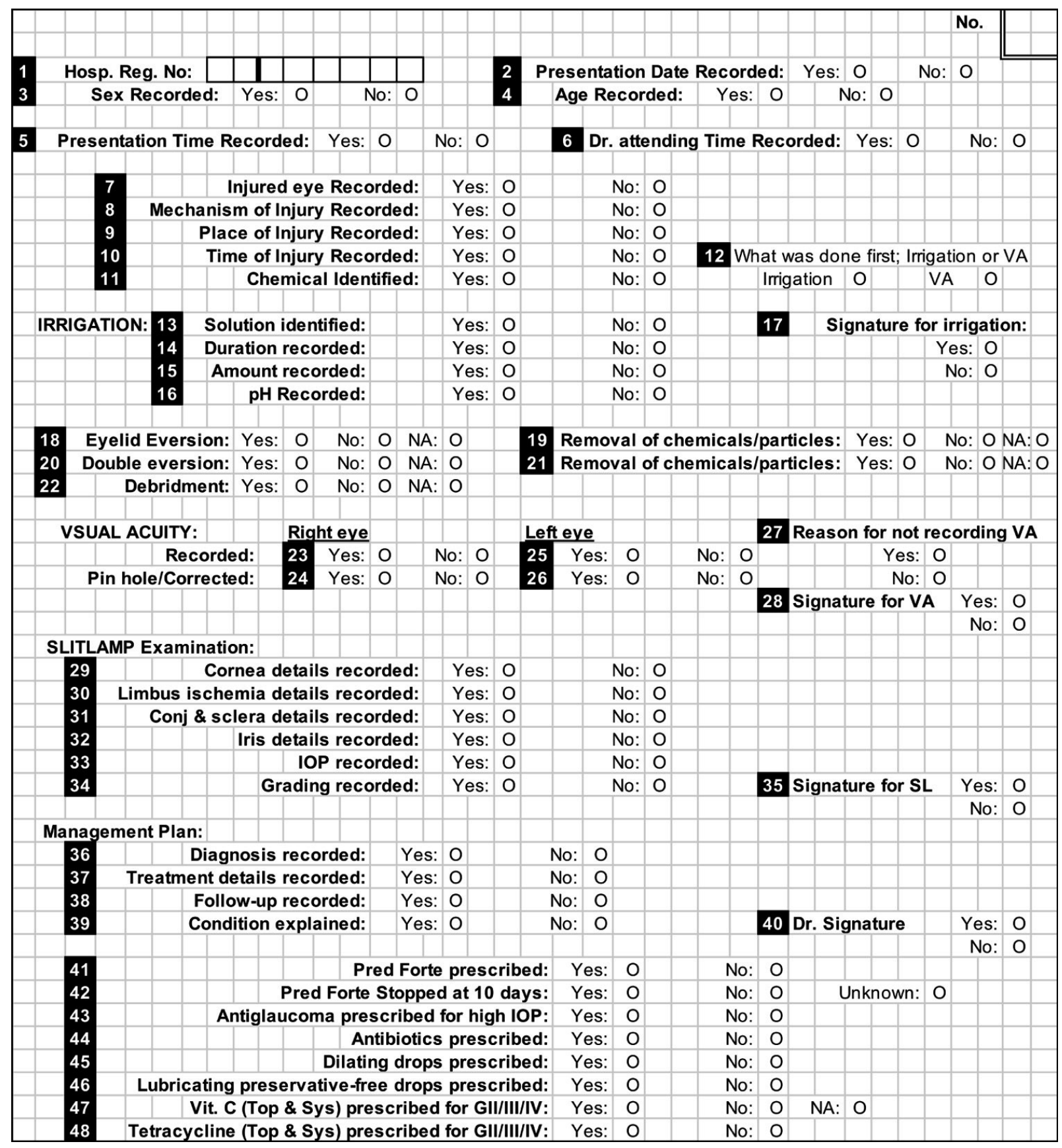

FIGURE 2. Structured questionnaire form

- lids: eyelid eversion, removal of chemical/particles, double eversion, debridement;

- visual acuity (VA): right and left eye VA and corrected VA, the reason for not recording VA, a signature of the person taking VA;

- slit-lamp examination (SLE): cornea details, limbus ischemia, conjunctiva and sclera details, iris details, intraocular pressure (IOP), grading, a signature of the person performing SLE;
- management plan: diagnosis, plan, follow-up, condition explained to the patient, attending physician signature;

- medication: steroid drops, information if a patient was advised to stop steroids at 10 days, anti-glaucoma drops for high IOP, antibiotic drops, dilating drops, lubricating drops, vitamin $\mathrm{C}$ (topical and systemic) for grades II/III/IV acid injury prescribed, tetracycline (topical and systemic) for grades II/III/IV prescribed. 
Questions had "yes" or "no" checkboxes. Data were stored and analysed using Excel charts.

\section{Method of literature search}

The literature search was performed using the online electronic PubMed. The keywords searched included: "eye burns", "ocular burns", "ocular chemical burns", "ocular chemical injuries", "chemical injuries of eye", "chemical injuries of ocular surface". Combinations of these terms were used as well. Relevant articles were reviewed. All English-language articles published between 2000 and 2020 were included.

All reported studies discussed chemical injury management. We found no studies related to the actual review of the assessment of emergency procedures and documentation. To the best of our knowledge, our study is the first to address this issue.

\section{RESULTS \\ Demographic data}

We found that the presentation date and time for patients were recorded in more than $90 \%$ of cases, and the attending time of the caregiver was recorded in $90 \%$ of cases. Sex and age for patients were recorded in $97.5 \%$ of cases.

\section{Injury data}

The injured eye (right or left) was recorded in $95 \%$ of cases. The mechanism of injury was recorded in $65 \%$ of cases. The time and place of injury were recorded in $55 \%$ and $10 \%$ of cases, respectively. The type of offending chemical was identified in $65 \%$ of cases.

\section{Irrigation data}

The amount of irrigating solution used was not recorded in any case, while the irrigation duration was recorded in $55 \%$ of cases. The irrigating solution was identified in $50 \%$ of patients, while $\mathrm{pH}$ was recorded in $52.5 \%$ cases. From the records, we could not determine if irrigation was performed before VA was taken.

\section{Debridement data (eversion, removal of particulate matter, and debridement)}

The eyelid eversion/double eversion procedures were recorded in $5 \%$ and $2.5 \%$ of cases, respectively. Removal of chemical or particulate matter and debridement were recorded in $15 \%$ of cases. It is presumed that these procedures were not per- formed because they were not recorded, and there was no documentation that the procedures were not required.

\section{Visual acuity data}

Visual acuity was recorded in both eyes in almost $90 \%$ of cases, while corrected VA was recorded in both eyes in nearly $50 \%$ of cases. The reason for not recording VA was recorded in $7.5 \%$ of cases.

\section{Slit-lamp examination data}

Details of cornea, conjunctiva, sclera, and iris details were recorded in $100 \%$ of cases. Limbal ischemia was documented in $45 \%$ of patients, and intraocular pressure was recorded in $25 \%$. Grading was recorded in $77.5 \%$ of cases.

\section{Management plan data}

The diagnosis was recorded in $100 \%$ of cases, while the management plan was recorded in 95\% of cases. Explanation of condition to patients was documented in $45 \%$ of cases, and follow-up time was recorded in $67.5 \%$ of cases.

\section{Medication data}

Antibiotics and steroids (drops/ointment) were prescribed in $92.5 \%$ of cases. Advising the patient to stop steroids on the 10th day was recorded in $15 \%$ of cases. Anti-glaucoma medication was not prescribed in any case. Lubricating drops were documented in $95 \%$ of cases, and dilating drops were prescribed in $7.5 \%$ of cases. Vitamin C and tetracycline medications for grades II, III, VI were prescribed in $12.5 \%$ and $7.5 \%$ of cases, respectively.

\section{Signatures}

Irrigation procedure signatures were recorded in $20 \%$ of cases, and visual acuity measurement signatures were documented in $100 \%$ of cases. Slit-lamp examination signatures were recorded in $75 \%$ of cases, and management plan signatures were documented in $95 \%$ of cases.

Figure 3 shows a clustered bar chart of all data collected in this study, including demographic data, injury, irrigation, debridement, VA, SLE, management plan, and medication.

\section{DISCUSSION}

The descriptive analysis of this study showed that the documentation for demography and VA parameters were not $100 \%$ for all parameters. Our 


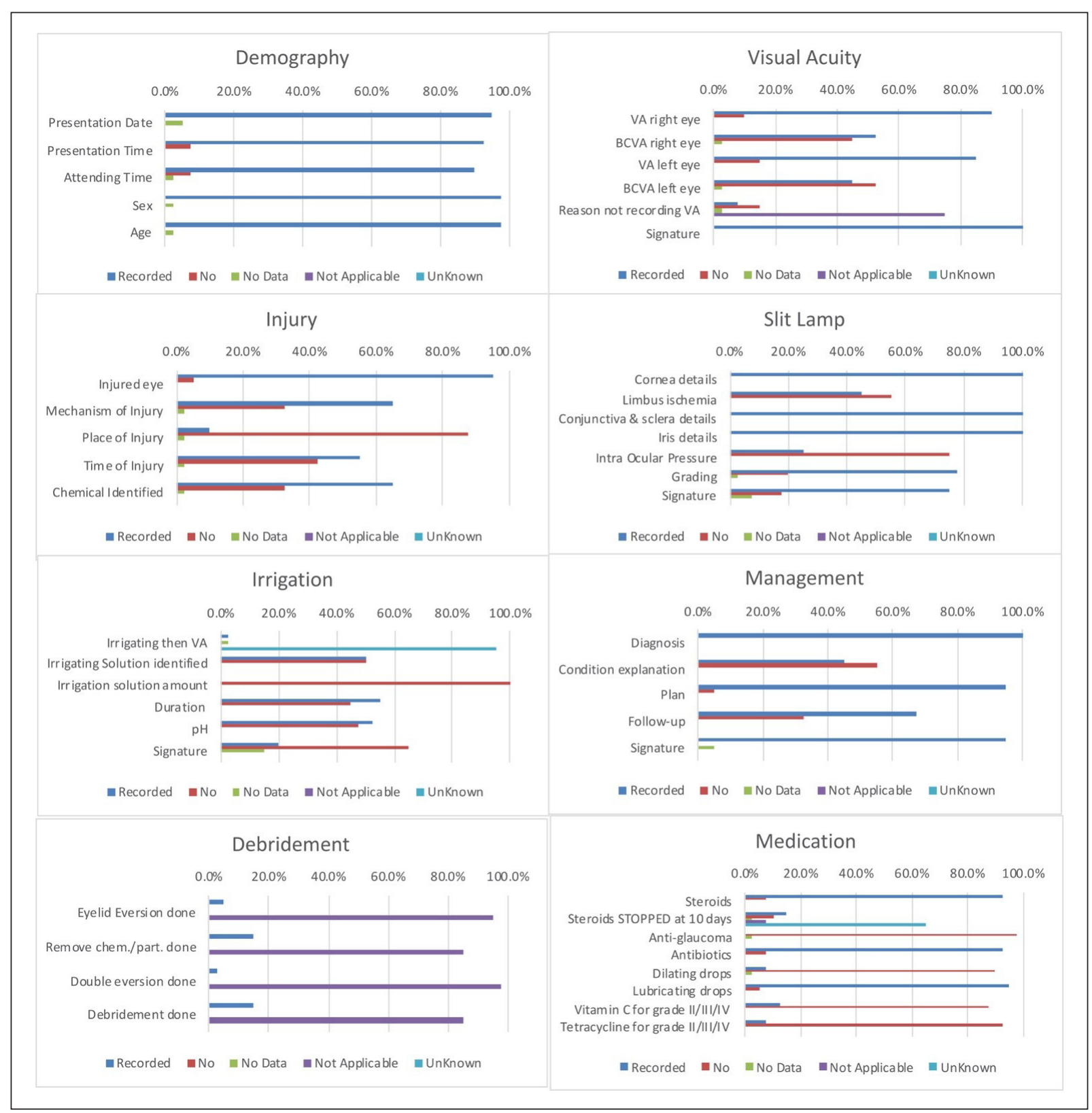

FIGURE 3. Results of the study

protocol calls for $100 \%$ documentation for all parameters. For each case where VA was not recorded, a reason should be documented. The same analysis was seen for the injury category, where none of the parameters were documented in $100 \%$ of cases. Our protocol calls for $100 \%$ documentation for all parameters.

With respect to the irrigation category, this study did not show caregivers' adherence to our protocol for chemical injury management. We could not tell which action was taken first, VA or irrigation. The type of irrigating solution, amount, duration of irrigation, and $\mathrm{pH}$ should be documented in each case.
Documentation of the debridement category showed nonadherence of caregivers to our protocol for chemical injury management. Lid eversion, double eversion, removal of chemical particles, and debridement should be performed on all chemical injury patients regardless of grade.

Regarding the SLE category, some parameters should be recorded in all cases. Such parameters include limbus ischemia details, intraocular pressure, and grading.

While documenting management showed that caregivers followed our protocol, it must be emphasized that caregivers should, in all cases, document 
the explanation of the condition to patients and their follow-up.

The results indicate that our caregivers followed the chemical injury protocol for the medication category in most cases. One parameter that must be documented in more detail is the advice to patients to stop steroids on the $10^{\text {th }}$ day. While the parameters of vitamin $\mathrm{C}$, tetracycline, and anti-glaucoma medications were not filled in most cases, it is assumed that these medications were not needed nor prescribed.

Documentation of signatures showed that our caregivers must sign on all procedures implemented.

Documentation records patient management, which includes admission, diagnosis, treatment plan, and medications already dispensed. Medical records are essential for continuing care for the patient by all health providers. Patients may also seek treatment at other facilities for follow-up. Accurate medical documentation ensures that any health provider will treat the patient correctly and prevents incorrect treatment. A treatment plan helps in facilitating that the patient receives the treatment needed for a full recovery.

Good medical documentation is critical in emergencies. Doctors need to know about a patient's diagnosis and underlying conditions to ensure that emergency treatments offered don't cause harm to the patient.

Documentation helps the medical facility ensure that the quality of care offered is up to standards.

In addition to the clinical importance of medical records, they are also a legal document that acts as evidence of the care provided. An incomplete medical history can adversely affect the quality of care for patients and leads to errors. Incomplete medical records show that care was incomplete and exhibited non-compliance with organizational protocols and policies. These incomplete records may result in legal actions and can cause a loss of revenue.

\section{CONCLUSIONS}

The results of this study reveal that our documentation needs improvement for several parameters. Several recommendations were formulated:

Emphasize to caregivers that irrigation must be done first.

Corrected visual acuity should be attempted for both eyes in all cases, and reasons for not recording it should be documented.

It is important to document and record limbus details, iris details and measure intraocular pressure in all cases.

\section{REFERENCES}

1. Hughes WF. Alkali burns of the eye; clinical and pathologic course. Arch Ophthal. 1946; 36: 189-214, doi: 10.1001/archopht.1946.00890210194005, indexed in Pubmed: 20997671.

2. Lubeck D, Greene JS. Corneal injuries. Emerg Med Clin North Am. 1988; 6(1): 73-94, indexed in Pubmed: 3278888.

3. Cohen KL, Hyndiuk RA. Ocular emergencies. Am Fam Physician. 1978; 18(4): 178-184, indexed in Pubmed: 707268.

4. Rodeheaver GT, Hiebert JM, Edlich RF. Initial treatment of chemical skin and eye burns. Compr Ther. 1982; 8(5): 37-43, indexed in Pubmed: 7094559.

5. Roper-Hall MJ. Thermal and chemical burns. Trans Ophthalmol Soc UK. 1965; 85: 631-653, indexed in Pubmed: 5227208.

6. Lin $M$, Ekşioğlu Ü, Mudumbai $R$, et al. Glaucoma in Patients With Ocular Chemical Burns. Am J Ophthalmol. 2012; 154(3): 481-485. e1, doi: 10.1016/j.ajo.2012.03.026, indexed in Pubmed: 22633350.

7. Wagoner MD. Chemical injuries of the eye: current concepts in pathophysiology and therapy. Surv Ophthalmol. 1997; 41(4): 275-313, doi: 10.1016/s0039-6257(96)00007-0, indexed in Pubmed: 9104767.

8. McCulley JP. Chemical injuries. In: Smolin G, Thoft RA. ed. The Cornea: Scientific Foundation and Clinical Practice. Little Brown and Co, Boston 1987: 527-42.

9. American Academy of Ophthalmology 2019, Chemical (Alkali and Acid) Injury of the Conjunctiva and Cornea. https://eyewiki.aao.org/ Chemical_(Alkali_and_Acid)_Injury_of_the_Conjunctiva_and_Cornea (June $17,20 \overline{2} 1)$.

10. The Royal College of Ophthalmologists 2019. Chemical injuries of the ocular. https://www.rcophth.ac.uk/wp-content/uploads/2018/04/ College-News-April-2018-Focus.pdf (June 17, 2021). 